\title{
Electrical Conductivity of Bone Marrow Cell Cultures and Their Connection with Proliferative Activity: Age Aspect
}

\author{
Valentyn Kozheshkurt', Anatoly Bozhkov ${ }^{2, *}$, Svetlana Ohiienko, \\ Anastasia Bondar ${ }^{2}$, Victor Katrych ${ }^{1}$, Anna Novikova ${ }^{2}$, Yevhenii Antonenko ${ }^{1}$, \\ Stanislav Haiovyi ${ }^{2}$ and Yurii Kot ${ }^{3}$
}

${ }^{I}$ Department of Physical and Biomedical Electronics and Complex Information Technologies, V. N. Karazin Kharkiv National University, Kharkiv, Ukraine

${ }^{2}$ Research Institute of Biology, V. N. Karazin Kharkiv National University, Kharkiv, Ukraine

${ }^{3}$ Department of Biochemistry, V. N. Karazin Kharkiv National University, Kharkiv, Ukraine

("Corresponding author’s e-mail: bozhkov@univer.kharkov.ua)

Received: 12 May 2020, Revised: 19 March 2021, Accepted: 26 March 2021

\begin{abstract}
Functional activity is a basic concept of biology; however, instrumental methods of its determination are absent. The interrelation between bone marrow cell proliferative activity, as one of the functional activity parameters, the content of calcium, copper, and reactive oxygen species (ROS) in the cells, and the electrical conductivity of the cell culture was studied in young and old Wistar rats aged 3 and 20 months. We determined the electrical conductivity of the bone marrow cell cultures and parameters of bone marrow cells (proliferative activity and calcium, copper and ROS content). The bone marrow cells of old intact animals had higher proliferative activity in the primary culture as compared to that of young animals. The content of the 8 main morphological cell types in the young and old animals were similar, while the lymphocyte content was larger in the old animals. Content of ions in the bone marrow cell cultures of old animals as compared with that in young animals varied as follows: Content of calcium was $75 \%$ higher, copper was 5 times lower, and ROS was 2.5 times lower. It was found that the conductivities of the pure culture medium and of that after the cultivation of the bone marrow cells in the nutrient medium were significantly different. It has been suggested that the electrical conductivity of biological media could be used as an integral characteristic of the ionic composition and structural organization of biological media.
\end{abstract}

Keywords: Bone marrow cells, Calcium, Electrical conductivity, Proliferative activity

\section{Introduction}

Investigation of the cell functional activity of various tissues and organs is of great interest in solving a number of medical and biological problems. First of all, it of concern to problems related to oncopathology, autoimmune pathology, gerontology, and geriatrics. However, researchers are faced with the fact that the concept of "functional state", which is a basic concept of modern science of biological systems, does not have an unambiguous interpretation. This is due to the lack of our knowledge about the integrative activity of biological systems [1]. In this regard, the search for instrumental methods for assessing functional states is an urgent task. In medical practice, when determining functional states, the concepts of "norm" and "pathology" are used. However, there is no borderline between a normally functioning system and pathology. At the same time, even minor deviations from the homeostatic norm can lead to persistent functional changes in response to subsequent actions of stress factors. Agedependent changes in biological systems can serve as an example of such functional changes. An example of functional changes at the cellular level of the organization of biological systems can be the activity of proliferation; that is, populations of non-dividing cells and actively dividing cells that can be considered as different functional states.

A number of works have been devoted to studying cell proliferative activity [2-4]. Significant successes have been reported in solving this vital problem $[5,6]$. It was shown that cell division regulation depends on a number of factors, and can be implemented in various ways [7]. Thus, there exists a need to search and develop integrative methods for assessing proliferative cell activity as a parameter of 
functional states. The available data suggest that integrative methods can be based on physical quantities, such as the electrical conductivity and permittivity of biological media. As is known, electrical conductivity is defined by charged particles moving in an electric field [8,9]. Data exist that indicate a relationship between the ionic status of cells and their proliferative activity [10]. In this regard, the identification of the relationship between the electrical conductivity of bone marrow cells (BMCs) and the functional activity of the cells is of great interest.

As is known, blood and immunocompetent cells are formed in the bone marrow. The functional activity of BMCs represents the physiological characteristics of the organism, in particular, pathologies and age-related changes. In this regard, the study of such integral characteristics as the proliferative activity of BMCs in culture and their relation with electrical conductivity is of great theoretical and practical interest.

Investigation of the electrical conductivity of biological objects was started 70 - 80 years ago in the $20^{\text {th }}$ century [11]. However, interest in this promising method was somewhat lost due to the difficulty of interpreting the obtained results. Our investigations have shown that the electrical conductivity of biological systems is affected not only by the ionic composition, but also by the presence of lipids and membrane structures, and the ratio between high and low molecular weight proteins in biological systems [12]. The conductivity index can reflect the peculiarities of the organization of biological systems [13]. In this regard, it was hypothesized that the electrical conductivity of biological systems depends on the number of charged molecules and the structural organization of the systems and, therefore, this indicator can be an integral indicator of the functional activity of biological systems.

\section{Materials and methods}

\section{Experimental approach}

To check the hypothesis, the BMCs of Wistar rats of different ages (3 months as young and 20 months as old animals) were obtained. Then, the proliferative activity of the BMCs in culture was defined in vitro and the electrical conductivity of the BMC cultures was measured. The content of calcium and copper ions, which are present in relatively large amounts in the cells, and the reactive oxygen species, known as indicators of the redox system in the BMCs, were determined in the young and old animals. These parameters indicated the functional activity of the BMCs.

The experimental study was based on the assumption that formation of adaptive memory affects all hierarchical levels, starting from physicochemical to cellular results, in the temporal existence of biological systems. Therefore, the BMCs of young and old animals with different adaptive experience must differ in characteristics of the ionic composition, redox system, and functional cell properties. Thus, it was expected that the BMCs formed in young and old animals from mesenchymal stem cells on the background of different microenvironments should differ in physicochemical and functional characteristics.

To assess some physicochemical characteristics of the BMCs, the parameters of the contents of copper, an essential trace element of more than 30 enzymes, calcium, and reactive oxygen content (ROS) were determined. The electrical conductivity of the BMC cultures isolated from young and old animals was also measured as an integral indicator of ion transport activity in an electric field.

\section{Preparing animals for the experiment}

The experiments were carried out in sexually mature young and old male Wistar rats, which were divided into 2 age groups ( 3 and 20 months), consisting of 6 animals. All procedures involving animals were carried out observing bioethics rules (Council Directive 2007/526/EC, 2007) [14] taking into account the circadian rhythms of biological responses. Therefore, animals were always fed according to a timetable and were kept in standard conditions, and all manipulations (the administration of toxic or other preparations) were carried out before their food intake. The animals did not receive any food for $24 \mathrm{~h}$ preceding BMC isolation. The animals were always withdrawn from the experiment from 8:00 to 10:00 a.m. local time.

\section{Extracting the BMCs and their cultivation in vitro}

The BMCs were extracted from 2 femoral rat bones following the procedure described in [15], and then were cultivated in Eagle's medium, which included antibiotics (1\% gentamycin and $1 \%$ streptomycin) and $20 \%$ of inactivated fetal bovine serum. Cultivation was run under the standard conditions: Temperature was $37^{\circ} \mathrm{C}$, and atmospheric $\mathrm{CO}_{2}$ content was $5 \%$. The number of cells and their morphotypes were determined daily from the first to the fourth day of cultivation. The content of the 
BMCs at the initial stage of cultivation always reached 2 million cells per $\mathrm{mL}$ (Figure 1), and was unchanged during cultivation.

Determining the number and morphotypes of the cells

The cell count and evaluation of the viability of the BMCs were carried out as described in [16]. The BMC morphotypes were determined immediately after the culture preparation, and on the $2^{\text {nd }}$ and $4^{\text {th }}$ day of cultivation, as was described in [17]. The cytological preparations were stained by the Romanovskii-Gimza method, and then were analyzed by using a 100× Zeiss Primo Star iLED microscope (Germany).

\section{Measuring the calcium and ROS contents}

The content of calcium ions in the BMCs was measured by adding $5 \mu \mathrm{L}$ of the working solution to the BMC cultures. Then, the components were mixed by pipetting and were incubated for $15 \mathrm{~min}$ in a dark place at room temperature. The cells were visualized by confocal microscopy using an Olympus FV10i-LIV laser scanning confocal microscope (Olympus, Japan) with the Olympus FV4.1 software. The relative intensity of fluorescent radiation in each cell was measured by the Olympus FV4.1 software, taking into account the background glow. The image deconvolution and 3D object reconstruction was carried out by using Olympus CellSense Dimension Desktop software. The ROS content was measured according to the recommendations of the manufacturer (Cellular ROS Assay Kit - Red (ab186027) [18] by adding $50 \mu \mathrm{L}$ of the cell culture $\left(6 \times 10^{6} / \mathrm{mL}\right)$ to $5 \mu \mathrm{L}$ of Fluo-3 to working solution with a content of 25 $\mu \mathrm{M}$.

\section{Determination of content of copper ions}

The copper ions in the BMC cultures were determined by adding a mixture of sulfuric and nitric acids $\left(1 \mathrm{~mL}\right.$ of each acid) to $500 \mathrm{~mL}$ of the cultures. Then, the samples were heated at $140{ }^{\circ} \mathrm{C}$ for $60 \mathrm{~min}$ for mineralization. The content of copper ions in $\mu \mathrm{g} / 10^{6}$ cells was determined by using an atomic adsorption spectrophotometer C-115-M1 (Ukraine).

\section{Measuring the electrical conductivity of the BMCs}

The BMCs were suspended in the culture medium, consisting of 199 media with antibiotics and $20 \%$ fetal calf serum, so that $1 \mathrm{~mL}$ contained 3 million cells $/ \mathrm{mL}$. The cultures were placed into a Teflon cell, connected to 2 ports of the Rohde \& Schwarz ZNB 40 vector analyzer. The electrical conductivity was measured in the frequency range from $100 \mathrm{kHz}$ to $100 \mathrm{MHz}$ with intervals of $50 \mathrm{kHz}$.

The S-parameters of measuring cell, real and imaginary parts of the impedance, $Z_{r e}$ and $Z_{i m}$, were automatically measured by using a Rohde \& Schwarz ZNB 40 analyzer. To calculate the electrical conductivity of the cell cultures, the measuring cell impedance was converted to the equivalent parameters of an electric circuit: A capacitor and resistor connected in parallel. Then, capacitance $C$ and resistance $R$ of the equivalent circuit could be expressed through the real and imaginary parts of the impedance as:

$$
\begin{aligned}
& C=-\frac{1}{\omega \cdot\left(\frac{Z_{r e}^{2}+Z_{i m}^{2}}{Z_{i m}}\right)}, \\
& R=Z_{r e}+\frac{Z_{i m}^{2}}{Z_{r e}}
\end{aligned}
$$

Since the electrical conductivity of the measuring cell contents depended upon the properties of the substance in the measuring cell and its geometric parameters, the adjustment factor $k=175 \mathrm{~m}^{-1}$ calculated for a cylindrical cell with a capacity of $1 \mathrm{~mL}$ was introduced. Then, the specific conductivity $\sigma$ was defined as:

$$
\sigma=k \cdot \frac{1}{R} .
$$




\section{Statistical analysis of the results}

The mean, standard deviation, and standard error of the mean, and sample size were used as the characteristics of the obtained samples. The statistical significance of difference between the 2 data groups was evaluated by the nonparametric Mann-Whitney test. The results were statistically processed by the OpenOffice and Origin software packages. The data differences between the control and experimental groups were accepted as significant at $\mathrm{p}<0.05$.

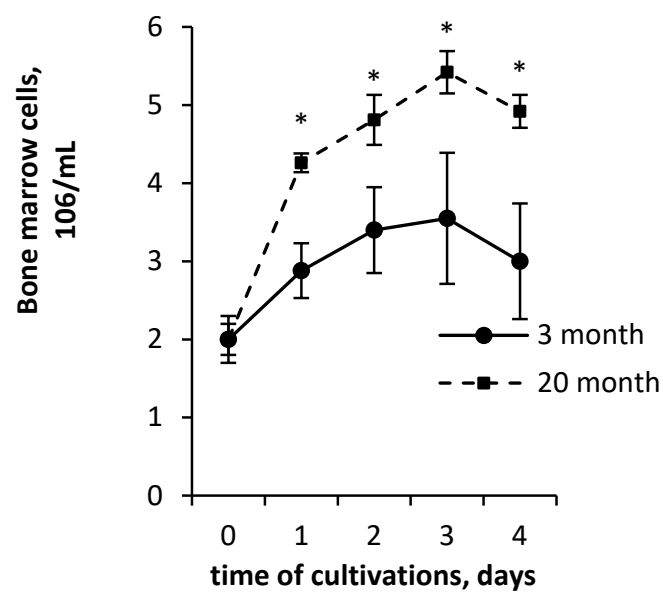

(a)

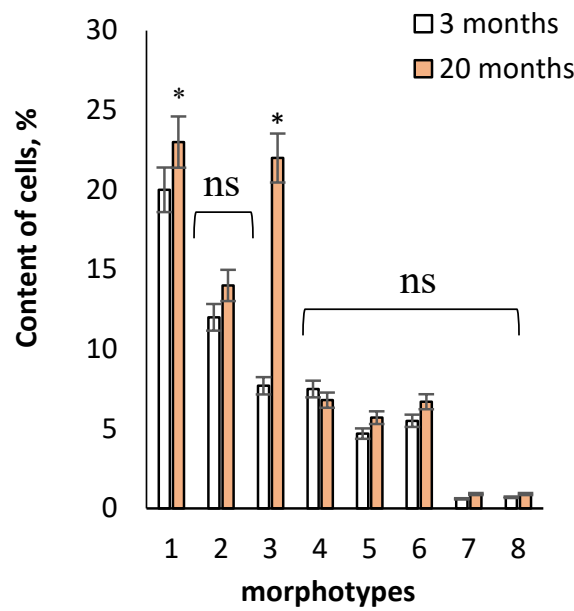

(b)

Figure 1 The characteristics of the cell culture: a) the number of BMCs in primary culture obtained from young and old intact animals; b) the number of morphologically identified morphotypes (1 - stab neutrophils, 2 - metamyelocytes; 3 - lymphocytes, 4 - segmented neutrophils, 5 - myelocytes, 6 eosinophils, 7 - basophils, 8 - monocytes) obtained from young and old animals. All the experiments were repeated as biological replicates for at least 3 times. The differences between samples were analyzed using the nonparametric Mann-Whitney $\mathrm{U}$ test. Data are shown as mean \pm S.D. ${ }^{*} p<0.05$; ns, not significant.

\section{Results and discussion}

\section{Proliferative activity of the BMCs in young and old animals in vitro}

The BMCs obtained from old animals were transferred to the primary culture. In a day, the cell number increased from 2 to 4 million cells $/ \mathrm{mL}$. By the $3^{\text {rd }}$ day, the number of BMCs reached a steadystate at a level of more than 5 million cells $/ \mathrm{mL}$ (Figure 1). The BMCs of young animals were also proliferated in the culture. However, their growth rate was less than that of old animals (Figure 1a).

Thus, the number of BMCs obtained from the old animals after 1 day of cultivation was $47 \%$ higher as compared to that of the young animals. Such behavior was observed during the whole cultivation period (Figure 1a).

Hence, the proliferative potential of the BMCs of old animals, placed into primary culture, was greater than that of young animals. This observation may seem unusual. However, the obtaining of BMCs and their further cultivation was accompanied by an abrupt change in the microenvironment of these cells. It cannot be ruled out that BMCs obtained in old animals respond to changes in the environment by increasing proliferative activity to a greater extent than that of the BMCs of young animals. Along with this, it is known that not all types of BMC are capable of proliferation in culture in comparison with the BMC of young animals. In this regard, in the next series of experiments, the number of morphologically identified types of the BMCs in young and old animals was determined. The 8 main morphotypes of the BMCs: Monocytes, myelocytes, metamyelocytes, stab and segmented neutrophils, lymphocytes, basophils, and eosinophils, represent $60-80 \%$ of all BMC types [18].

The BMC composition of young and old animals has been proven to be similar, with the exception of lymphocytes (Figure 1b). The number of lymphocytes in the BMCs of old animals was 2.8 times greater as compared with that of young animals (Figure 1b). An increase in the number of lymphocytes in the bone marrow of old animals may reflect the formation of a leukemic clone in old animals, which may be associated with the onset of the development of a pathological process in these animals. Since 
lymphocytes are capable of proliferation in cell culture, and their number is increased in old animals, it can be assumed that the high proliferative activity of the BMC culture obtained in old animals is explained by the large number of lymphocytes in the BMCs of old animals. At the same time, it cannot be ruled out that the BMCs formed in old and young animals against the backgrounds of different microenvironments would differ in physicochemical characteristics affecting their functional activity, such as proliferation. Based on this assumption, the ion composition and ROS content of the BMCs were determined in the following series of experiments.

\section{The ion composition and ROS content of the BMCs in young and old intact animals}

The content of calcium ions in the BMCs of the old rats was $73 \%$ greater than in those of young one. The content of copper ions was 5 times lower in the young rats (Table 1).

Table 1 The content of copper, calcium, and ROS in the BMCs of young and old animals $(n=6)$. Reliable differences with $p \leq 0.05$, obtained by the nonparametric Mann-Whitney U test, are noted by *

\begin{tabular}{ccc}
\hline \multirow{2}{*}{ Element } & \multicolumn{2}{c}{ Mean \pm error of the mean } \\
\cline { 2 - 3 } & Young animals & Old animals \\
\hline $\mathrm{Cu}^{2+}, \mu \mathrm{g} / 10^{6}$ cells & $0.072 \pm 0.005$ & $0.013^{*} \pm 0.002$ \\
$\mathrm{Ca}^{2+}$, relative units & $167 \pm 4.9$ & $230^{*} \pm 8.1$ \\
ROS relative units & $374 \pm 7.6$ & $148^{*} \pm 5.3$ \\
\hline
\end{tabular}

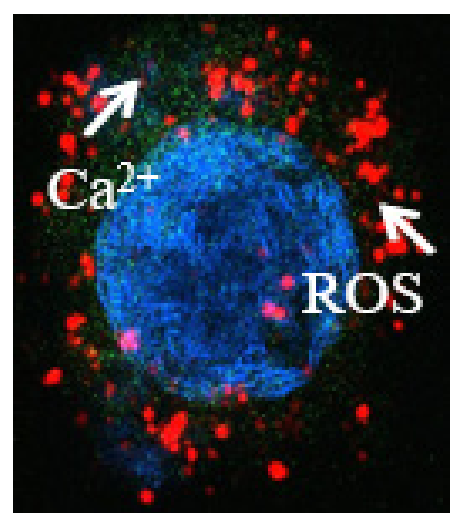

Figure 2 The content of $\mathrm{Ca}^{2+}$ and ROS were measured by an Olympus FV10i-LIV laser scanning confocal microscope with Fluo-3 and Cellular ROS Assay Kit - Red (ab186027), respectively. The relative intensity of fluorescent radiation of $\mathrm{Ca}^{2+}$ and $\mathrm{ROS}$ in each cell was measured by the Olympus FV4.1 software. Localization of calcium ions and ROS in bone marrow cells was measured. All the experiments were repeated as biological replicates for at least 3 times. The differences between samples were analyzed using the nonparametric Mann-Whitney U test. Data are shown as mean \pm S.D. ${ }^{*} p \leq 0.05$.

As is known, the ionic composition of cells is regulated by various mechanisms affecting the functional activity of the BMCs [19]. Metal ions are part of a large number of enzymes which regulate cell activity [20], provide intracellular signaling [21], regulate intracellular transportation, and affect the characteristics of the cell redox system.

The ROS in the BMCs of old animals was 2.5 times lower than in that of young animals (Table 1 and Figure 2). These results suggest that the equilibrium of the prooxidant - antioxidant system in the BMCs of old animals is shifted towards antioxidants, while that of young animals is shifted toward prooxidants.

Consequently, the bone marrow of old animals contained a significantly larger number of lymphocytes compared to the number of lymphocytes in the bone marrow of young animals. The number of all other morphotypes in the bone marrow of young and old animals was the same. The transfer of cells 
to primary culture was accompanied by an increase in the proliferative activity of BMCs isolated from old animals.

It is known that calcium ions are involved in the regulation of the functional activity of cells, including proliferative activity. However, this relationship is quite complex, in the sense that a number of works have revealed both positive and negative relationships. This can be explained by the fact that a change in the number of some ions is accompanied by a change in the number of other ions. The relationship between ions in a cell is determined by a complex set of integrative interactions. In this regard, it is of interest to search for methods for assessing the total ionic composition of a cell. It is known that the ionic composition in complex biological media can be determined by the characteristics of the electrical conductivity of biological systems.

\section{The electric conductivity of the BMCs in young and old intact animals}

The electric conductivity of the BMC cultures obtained from old animals was significantly higher than that of young animals (Figure 3). It is known that electrical conductivity in biological systems is determined by the content of ions in solution. However, the electrical conductivity will be affected by the structure of cell membranes, the content of lipoproteins, and the state of high and low molecular weight proteins that make up the cells. Currently, there is no unified theory of the electrical conductivity of biological systems that would determine the contribution of each of these components to the formation of electrical conductivity. The obtained differences in the electrical conductivity of the BMC suspension of young and old animals corresponded to the differences in the proliferative activity of these cells (Figures 1a and 3a).

Therefore, the similar morphotypes in the BMCs of the young and old animals differing in the ionic composition, ROS content, and proliferation ability in primary culture may indicate their functional differences within the same morphological types.

The electrical conductivity of the cell suspension was selected as an integral characteristic of the ionic composition.

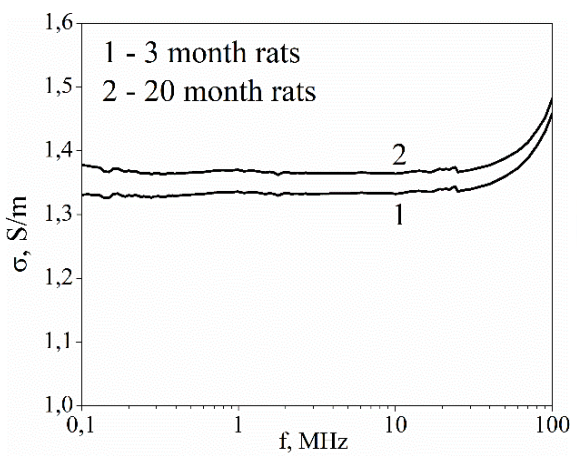

(a)

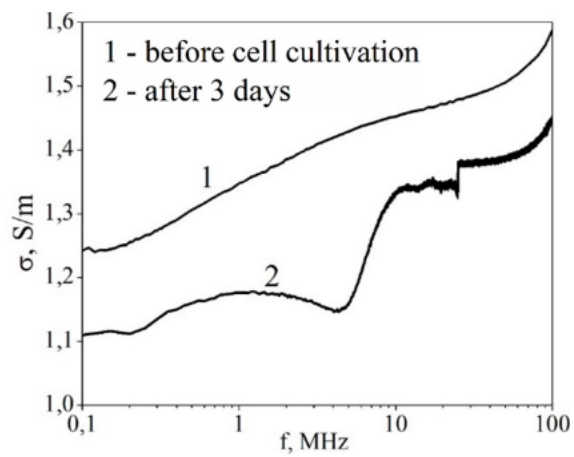

(b)

Figure 3 The frequency plots of the electrical conductivity of the BMC cultures: a) 3 month rats (1) and 20 month rats (2); b) the culture medium before cell cultivation (1) and after 3 days of cell cultivation after the removal of cells from the medium (2).

It should be noted that the frequency curves of the electrical conductivity of the BMC cultures of young and old animals differed over the entire frequency range (Figure 3a). Since the culture of the BMCs from young and old animals contained the same number of cells ( 3 million cells $/ \mathrm{mL}$ ), it can be stated that the cellular structures of the old animals contained a larger number of charged particles and, additionally, the cell functional activity of young and old animals also differed.

It was previously shown that, along with the ionic composition, the parameters of electrical conductivity are significantly influenced by lipid components and the proteins composition [12]. Thus, the removal of lipid components from the medium was accompanied by an increase in electrical conductivity. Removal of high molecular weight proteins from a measuring cell was accompanied by a twofold increase in electrical conductivity. It should be noted that lipid and protein components have different effects on electrical conductivity. While lipid components are dielectrics, then high-molecular- 
weight proteins, the removal of which is accompanied by an increase in electrical conductivity, have a system-forming function. These results allow us to assert that, in multicomponent, complex biological systems, electrical conductivity depends both on the number of ions and other charged molecules, and on the structural organization of biological systems and the presence of molecules with dielectric properties. In this regard, the electrical conductivity of biological systems is an instrumental indicator of their structural and functional organization. This hypothesis can be supported by the data on the electrical conductivity of the culture media before and after the cultivation of bone marrow cells.

It was found that the electrical conductivity of the culture medium without cells was lower than that of the cell cultures. As can be seen from Figure 3b, the electrical conductivity of the pure culture medium is an almost linearly increasing function of the frequency. This may be explained by the fact that the capacitive component of the impedance increases as the frequency is increased due to presence of components with dielectric properties, since the culture medium is a mixture of compounds with different electrical properties.

If the cells were cultured for 3 days in the medium, and then were removed, the electrical conductivity of such a medium would be significantly lower than that of the pure medium. Such a decrease in the electrical conductivity of the medium after 3 days of cell growth indicates a significant decrease in the content of ions and charged molecules, capable of providing electric current conduction, and an increase in components with dielectric properties in such a medium. These are components, in particular lipoproteins, secreted by cells during cultivation. The fact of a change in the culture medium composition is also confirmed by the fact that for such a medium the interrelation between the electrical conductivity and the frequency of the electric current is revealed (Figure 3b).

These results indicate that medium composition during cell cultivation varies significantly, and the capacitive component of the measuring cell impedance becomes predominant. As is known, the cells in the process of cultivation release into the medium the amino acids, peptides, and fragments of nucleic acids, and absorb ions and other molecules. It is important to note that dynamically varying medium composition is accompanied by the formation of supramolecular complexes in medium. It is quite clear that dissolved molecules and supramolecular complexes have different electrical conductivity.

Earlier, the possibility of assessing the rate of metabolic processes in a cell by measuring changes of the electrical conductivity of the culture medium after the removal of cells was investigated. It was shown that the electrical conductivity of the medium depends on the physiological and chemical characteristics of cells [12].

Hence, the initial culture medium, the medium after cultivating the BMCs, and the culture of the BMCs in the culture medium significantly differed in electrical conductivity (Figure 3).

These differences cannot be explained only by the ionic composition of these media, but explanation should also include their structural organization: The presence of supramolecular complexes and dielectric elements such as membranes. We believe that the electrical conductivity of biological multicomponent dynamically changing media can serve as integral indicators of their characteristics.

Thus, the results may be summarized as follows: 1) the ionic composition of the culture medium changed after BMC cultivation for 3 days; 2) the electrical conductivity of biological media depends not only on the ionic composition, but also on its other components; 3 ) the proliferative activity of cells, their composition, and electrical conductivity are interrelated with one another; 4) the electrical conductivity of the cell suspension can be used in comparative studies and the evaluation of their functional activities; 5) the electrical conductivity of culture media can be an integral characteristic of the cells.

\section{Conclusions}

The results of this work allow us to conclude that the increased amount of calcium ions and the reduced number of free radical reaction products in the BMCs of old animals was accompanied by increased proliferative cell activity in the primary culture. The BMC suspension of the old animals can be characterized by increased electrical conductivity as compared to that of the young animals. The electrical conductivity of cell suspensions can be used as the integral indicator of proliferative cell activity. The data concerning the electrical conductivity of various biological media- the culture medium before and after cultivation, and also of the cell suspension- are of great interest.

Frequency characteristics of the electrical conductivity for various biological media allows us to state that several important conclusions can be drawn. First, biological media are multicomponent dynamic systems, in the sense that their supramolecular components can be composed and decomposed. Analytical analysis of biological media encounters great difficulties; therefore, biophysical methods can be more promising in searching for the integral indicators of biological media. Second, the electrical 
conductivity of biological media also depends upon components with dielectric properties, such as lipids and membrane structures. Intermolecular interactions, based on hydrophobic, hydrogen, and electrostatic interactions, can be established between membranes and other components of biological media, i.e., supramolecular structures can be formed which affect the electrical conductivity. Therefore, it can be assumed that the electrical conductivity of biological media is an integral characteristic of the electrolyte compositions and unexplored features of under molecular complexes. Assessment of the electrical conductivity of culture media can be used in cell biotechnology to estimate the quantitative parameters of culture media.

\section{References}

[1] A Mleiki, NT El Menif and I Marigómez. Integrative assessment of the biological responses elicited by metal pollution in the green garden snail, Cantareus apertus: Laboratory and field studies. Ecol. Indicat. 2020; 117, 106589.

[2] CE Lebert-Ghali, M Fournier, L Kettyle, A Thompson, G Sauvageau and JJ Bijl. Hoxa cluster genes determine the proliferative activity of adult mouse hematopoietic stem and progenitor cells. Blood. J. Am. Soc. Hematol. 2016; 127, 87-90.

[3] Maximilien Evrard, IWH Kwok, SZ Chong, KWW Teng, E Becht, J Chen, JL Sieow, HL Penny, GC Ching, S Devi, JM Adrover, JLY Li, KH Liong, L Tan, Z Poon, S Foo, JW Chua, IH Su, K Balabanian, F Bachelerie, SK Biswas, A Larbi, WYK Hwang, V Madan, HP Koeffler, SC Wong, EW Newell, A Hidalgo, F Ginhoux, LG Ng. Developmental analysis of bone marrow neutrophils reveals populations specialized in expansion, trafficking, and effector functions. Immunity 2018; 48, 364-79.

[4] CC Bain, CA Hawley, H Garner, CL Scott, A Schridde, NJ Steers, M Mack, A Joshi, M Guilliams, AMI Mowat, F Geissmann and SJ Jenkins. Long-lived self-renewing bone marrow-derived macrophages displace embryo-derived cells to inhabit adult serous cavities. Nat. Comm. 2016; 7, 11852.

[5] S Ullrich and R Guigó. Dynamic changes in intron retention are tightly associated with regulation of splicing factors and proliferative activity during B-cell development. Nucleic Acids Res. 2020; 48, 1327-40.

[6] BF Mogharbel, E Abdelwahid, AC Irioda, JC Francisco, RB Simeoni, DD Souza and KAT de Carvalho. Bone marrow-derived stem cell populations are differentially regulated by thyroid or/and ovarian hormone loss. Int. J. Mol. Sci. 2017; 18, 2139.

[7] J Lu, QH Wang, LH Huang, HY Dong, LJ Lin and JM Tan JM. Correlation of CDC42 activity with cell proliferation and palmitate-mediated cell death in human umbilical cord Wharton's jelly derived mesenchymal stromal cells. Stem Cell. Dev. 2017; 26, 1283-92.

[8] DA Dean, T Ramanathan, D Machado and R Sundararajan. Electrical impedance spectroscopy study of biological tissues. J. Electrostat. 20008; 66, 165-77.

[9] V Kozheshkurt, Y Antonenko, D Shtoda, O Slipchenko and V Katrych. Possibilities of impedance spectroscopy for the study of bioliquids. In: Proceedings of the $9^{\text {th }}$ International Conference on Ultrawideband and Ultrashort Impulse Signals, Odessa. 2018 p. 280-4.

[10] K Marycz, P Sobierajska, A Smieszek, M Maredziak, K Wiglusz and RJ Wiglusz. $\mathrm{Li}^{+}$activated nanohydroxyapatite doped with $\mathrm{Eu}^{3+}$ ions enhances proliferative activity and viability of human stem progenitor cells of adipose tissue and olfactory ensheathing cells. Further perspective of nHAP: $\mathrm{Li}^{+}, \mathrm{Eu}^{3+}$ application in theranostics. Mater. Sci. Eng. C Mater. Biol. Appl. 2017; 78, 151-62.

[11] JJ Ackmann and MA Seitz. Methods of complex impedance measurements in biologic tissue. Crit. Rev. Biomed. Eng. 1984; 11, 281-311.

[12] V Kozheshkurt, I Ivanov, Y Antonenko, V Katrich, A Bozhkov and T Gromovoy. Devising an express method for estimating the quality of colostrum and its components based on electrical conductivity. E. Eur. J. Enterprise Tech. 2021; 1, 69-77.

[13] X Bao, I Ocket, J Bao, J Doijen, J Zheng, D Kil, Z Liu, B Puers, D Schreurs and B Nauwelaers. Broadband dielectric spectroscopy of cell cultures. IEEE Trans. Microw. Theor. Tech. 2018; 66, 5750-9.

[14] Council Directive of 18 June 2007 on the approximation on guidelines for the accommodation and care of animals used for experimental and other scientific purposes (2007/526/EC). The Council of the European Communities, Brussels, 2007.

[15] SR Amend, KC Valkenburg and KJ Pienta. Murine hind limblong bone dissection and bone marrow isolation. J. Vis. Exp. 2016; 14, 53936. 
[16] AI Bozhkov, EG Ivanov, YA Kuznetsova, SL Ohiienko and AY Bondar. Copper-induced liver fibrosis affects the behavior of bone marrow cells in primary culture. Front. Boil. 2017; 12, 271-9.

[17] K Lewandowski, MM Kowalik, R Pawlaczyk, J Rogowski and A Hellmann. Microscopic examination of bone marrow aspirate in healthy adults-comparison of two techniques of slide preparation. Int. J. Lab. Hematol. 2012; 34, 254-61.

[18] Cellular ROS Assay Kit - Red (ab186027). Protocol booklet. Abcam, Cambridge, 2017, p. $2-9$.

[19] WTW Potts and G Parry. Osmotic and ionic regulation in animals: International series of monographson pure and applied biology. Pergamon Press, Oxford, 2016.

[20] HW Ryu, DH Lee, HR Won, KH Kim, YJ Seong and SH Kwon. Influence of toxicologically relevant metals on human epigenetic regulation. Toxicol. Res. 2015; 31, 1-9.

[21] C Blajszczak and MG Bonini. Mitochondria targeting by environmental stressors: Implications for redox cellular signaling. Toxicology 2017; 391, 84-9. 